\title{
Retinal findings in pediatric patients with Usher syndrome Type 1 due to mutations in MYOTA gene
}

\author{
Olaia Subirà ${ }^{1}$ Jaume Català-Mora ${ }^{1,2}$ - Jesús Díaz-Cascajosa ${ }^{2} \cdot$ Noel Padrón-Pérez $^{1}$ - M. A. Claveria ${ }^{2}$ • \\ Natalia Coll-Alsina ${ }^{2} \cdot$ Crystel Bonnet $^{3,4,5} \cdot$ Christine Petit $^{4,5,6,7} \cdot$ J. M. Caminal ${ }^{1} \cdot$ Joan $^{\text {Prat }}{ }^{2}$
}

Received: 12 September 2018 / Revised: 2 June 2019 / Accepted: 1 July 2019 / Published online: 18 July 2019

(c) The Author(s), under exclusive licence to The Royal College of Ophthalmologists 2019

\begin{abstract}
Purpose To describe retinal alterations detected by swept-source optical coherence tomography (SS-OCT) in paediatric patients with Usher syndrome type 1 (USH1) and to compare these findings to previously published reports.

Methods Thirty-two eyes from 16 patients (11 males and 5 females) with a genetic diagnosis of USH1 because of MYO7A mutations underwent SS-OCT. Patients ranged in age from 4 to 17 years (mean, 11,13 4,29). The subfoveal and macular area were analysed with SS-OCT at $1050 \mathrm{~nm}$ using 12 radial scans of $12.0 \mathrm{~mm}$. Structural abnormalities were evaluated and correlated with best-corrected visual acuity (BCVA).

Results The most common qualitative retinal abnormality was external layer damage in macular area. Specific alterations included external limiting membrane loss/disruption (27 eyes; 84.4\%), disruption of the Myoid zone (27 eyes; 84.4\%); Ellipsoid zone disruption (28 eyes; 87.5\%), and loss of the outer segments (29 eyes; 90.6\%). The damage of the retinal pigment epithelium was divided according to the loss of the different layers: phagosome zone (30 eyes; $93.8 \%$ ), melanosome zone (29 eyes; $90.6 \%)$ and mitochondria zone (0 eyes; $0 \%)$. The presence of cystoid macular oedema (CMO) was significantly correlated with alterations in photoreceptors. Disruption or absence of the myoid and ellipsoid zones of the photoreceptors were the only variables independently associated with decreased BCVA.

Conclusions The findings of this study suggest that the physiopathologic basis of early-stage Usher syndrome (USH) may be changes in the outer retinal layer, particularly the photoreceptors, which in turn may cause alterations-such as CMO-in the inner retinal layers. Accordingly, monitoring the condition of photoreceptors during follow-up may be advisable for the early detection of pathologic changes.
\end{abstract}

Olaia Subirà

osubira@bellvitgehospital.cat

1 Hospital Universitari de Bellvitge, L'Hospitalet de Llobregat, Barcelona, Spain

2 Hospital de Sant Joan de Déu, Esplugues de Llobregat, Barcelona, Spain

3 Syndrome de Usher et Autres Atteintes Rétino-Cochléaires, Institut de la Vision, Paris, France

4 Institut National de la Santé et de la Recherche Médicale, UMRS120 Paris, France

5 Sorbonne Universités, UPMC University Paris, Complexité du Vivant, Paris, France

6 Genetics and Physiology of Hearing Laboratory, Institut Pasteur, Paris, France

7 College de France, Paris, France

\section{Introduction}

Usher syndrome (USH) is an autosomal recessive disease characterized by a combination of sensorineural hearing loss (HL) and retinitis pigmentosa (RP) [1]. This syndrome accounts for $18 \%$ of all cases of RP [2]. USH is a clinically and genetically heterogeneous syndrome, as evidenced by the large number of disease-associated genes (eleven in total with the most frequently mutated genes being USH2A and MYO7A). USH can be classified into three different clinical types based on the specific genes involved and on the severity of HL. The characteristic symptom of RP is nyctalopia, caused by the loss of rod outer segments. In the earliest stages of USH, rod loss results in ring-shaped scotoma in the mid-periphery, which can progress to involve the periphery and the macula. In the final stage, damage to the cone photoreceptors induces central vision loss [3]. 
The first generation of optical coherence tomography (OCT) had low resolution and did not allow us to distinguish retinal microscopical structures. The improvement in the resolution of the OCT with the arrival of spectraldomain OCT or swept-source OCT (SS-OCT), provides the opportunity to observe in vivo all the retinal layers, corresponding with the histological studies [4]. The analysis of the integrity of the photoreceptors and their relationship with the retinal pigment epithelium (RPE) is of major importance to understand the pathogenesis of degenerative retinal diseases like the RP in USH.

The aim of this study was to use SS-OCT to identify the qualitative alterations present in the retina of paediatric patients with early stage $M Y O 7 A$-associated Usher syndrome type 1 (USH1); determining the precise location of the primary retinal damage and the associated pattern of progression was of particular interest. Secondarily, we sought to correlate these alterations with best-corrected visual acuity (BCVA).

\section{Methods}

\section{Patients and study design}

In this retrospective study, we analysed 32 eyes from 16 patients (11 males and 5 females; age range 4-17 years; median age, $11 \pm 4,29$ years) with a genetic diagnosis of MYO7A-associated USH1. Three pairs $(n=6)$ of the patients were brothers (Table 1).

A comprehensive medical history was taken to determine age at diagnosis of USH, the first symptoms at onset, and family history of USH.

A complete ophthalmologic examination was performed, including determination of BCVA (decimal fractions with Snellen equivalent), automated visual field, electroretinography and SS-OCT.

The diagnosis of RP was based on the presence of relevant symptoms together with fundoscopic features (as

Table 1 Demographic and clinical features of patients with USH

\begin{tabular}{ll}
\hline Characteristic & Value \\
\hline $\begin{array}{l}\text { Age (years) }(n=16) \\
\text { Mean } \\
\text { (median) }\end{array}$ & $11,13(11)$ \\
Sex & \\
Male & $11(68.75 \%)$ \\
Female & $5(31.25 \%)$ \\
$\begin{array}{l}\text { Visual acuity } \\
\text { Mean } \\
\text { (median) }\end{array}$ & $0.57(0.6)$ \\
\hline
\end{tabular}

diffuse retinal vessel attenuation and RPE pigmentary changes), OCT alterations and severe reduction of the ERG responses. In all cases, audiometry was performed prior to study enrolment to confirm bilateral congenital HL. The diagnosis of USH was confirmed by genetic analysis (Table 2), with all of the patients having either one homozygous or two compound heterozygous mutations.

Exclusion criteria were: (1) history of any other ocular disease (cataract, uveitis or glaucoma); (2) refractive error $>$ 5 diopters (due to its possible effect on choroidal thickness) [5]; and (3) prior ocular surgery (within 6 months prior to study enrolment).

The study was approved by the Research Ethics Committee at our hospital. All procedures were performed according to the hospital code of ethics and the study design adhered to the tenets of the Declaration of Helsinki. Informed consent was obtained from all patients and/or their parents.

\section{Swept-source optical coherence tomography}

We scanned the macular area of both eyes using a Triton SS-OCT (Topcon Corporation, Tokyo, Japan) at a $1050 \mathrm{~nm}$ wavelength and scanning speed of 100,000 A-scans/second using a high-definition set of twelve $12.0 \mathrm{~mm}$ radial cuts. Retinal foveal and choroidal thicknesses were automatically measured and presented as a topographic map with nine subfields as defined by the Early Treatment Diabetic Retinopathy Study style grid.

The SS-OCT images were closely evaluated to identify and describe any qualitative vitreoretinal alterations. For this purpose, the following structures were analysed: vitreous; inner limiting membrane (ILM), the presence of epiretinal membranes (ERMs), macular micropseudocysts (MPC) and cystoid macular oedema (CMO). With the aim of focusing on photoreceptor health and the relationship between photoreceptors and RPE, we used the nomenclature proposed by Cuenca et al. [4], based on morphological correlation between histology (using immunohistochemical methods) and OCT. Therefore, the terms used to identify the different segments of the photoreceptors are: external limiting membrane (ELM), Myoid zone, cone Ellipsoids (ISeZ) and Cone outer segment (OS). Whereas the RPE structures are: phagosome zone of the RPE (PhaZ), Melanosome zone of the RPE (RPEmel) and Mitochondria zone of the RPE (RPEmitz).

All scans were performed with dilated pupils (using cyclopentolate).

\section{Statistical analysis}

A descriptive analysis was performed. Measures of the central value (mean and median) and dispersion (standard 
Table 2 USH mutations in this cohort

\begin{tabular}{|c|c|c|c|}
\hline \multirow[t]{2}{*}{ Patient } & \multirow[t]{2}{*}{ Gene } & \multicolumn{2}{|l|}{ Mutations } \\
\hline & & Allele 1 & Allele 2 \\
\hline 1 & MYO7A & c. 3508 G > A, p.Glu1170Lys & c. $3508 \mathrm{G}>$ A, p.Glu1170Lys \\
\hline 2 & MYO7A & c. 3508 G > A, p.Glu1170Lys & c. 3508 G > A, p.Glu1170Lys \\
\hline 3 & MYO7A & c. 2215 G > T, p.Glu739* & c. $722 \mathrm{G}>$ A, p.Arg241His \\
\hline 4 & MYO7A & c. 4489 G > C, p.Gly1497Arg & Exon 46 deleted \\
\hline 5 & MYO7A & c.5944 G > A, p.Gly1982Arg & c.5944 G > A, p.Gly1982Arg \\
\hline 6 & MYO7A & c. $2283-1 \mathrm{G}>\mathrm{T}$ & c. 3594 C > A, p.Cys1198* \\
\hline 7 & MYO7A & c. $6321 \mathrm{G}>$ A, p.Trp2107* & c.6025delG, p.Ala2009Profs*32 \\
\hline 8 & MYO7A & c. 2914 C > T, p.Arg972* & c.4131dupT, p.Gly1378Trpfs*6 \\
\hline 9 & MYO7A & c. 5392 C > T, p.Gln $1798^{*}$ & c. 5392 C > T, p.Gln $1798^{*}$ \\
\hline 10 & MYO7A & c.999 T > G, p.Tyr333* & c.999 T > G, p.Tyr333* \\
\hline 11 & MYO7A & $\begin{array}{l}\text { c.2874_2878delCCAGG, p. } \\
\text { Gln959Glyfs*5 }\end{array}$ & $\begin{array}{l}\text { c. } 2874 \_2878 \text { delCCAGG, p. } \\
\text { Gln959Glyfs*5 }\end{array}$ \\
\hline 12 & MYO7A & $\begin{array}{l}\text { c. } 2874 \_2878 \text { delCCAGG, p. } \\
\text { Gln959Glyfs*5 }\end{array}$ & $\begin{array}{l}\text { c. } 2874 \_2878 d e l C C A G G, p . \\
\text { Gln959Glyfs*5 }\end{array}$ \\
\hline 13 & MYO7A & c.999 T > G, p.Tyr333* & c. 999 T > G, p.Tyr333* \\
\hline 14 & MYO7A & c.4297delC, p.Gln1433Serfs*116 & c.6025delG, p.Ala2009Profs*32 \\
\hline 15 & MYO7A & c.4297delC, p.Gln1433Serfs*116 & c.6025delG, p.Ala2009Profs*32 \\
\hline 16 & MYO7A & c.6_9dupGATT, p. Leu4fsAsp*39 & c.5573 T > C, p.Leu1858Pro \\
\hline
\end{tabular}

deviations [SD] and interquartile ranges [IQR]) were determined for continuous variables. Categorical variables were described as frequencies and percentages.

The Shapiro-Wilk test was performed to explore the distribution of the numerical data. Univariate and multivariate linear regression models were performed to assess the influence of the study variables on BCVA. All statistical tests were two-tailed. Statistical significance was set at $P<$ 0.05. All statistical analyses were performed using SPSS version 24.0 (SPSS Inc., Chicago, IL; USA).

\section{Results}

\section{Demographics}

A total of 16 patients (5 females [31.25\%] and 11 males [68.75\%]) were included. Of these, nine were of European origin, five were of South-East Asian origin and two of South-American origin. All patients had a genetic diagnosis of USH1, determined by the presence of the mutated gene MYO7A. (Table 2) We identified the disease-causing mutations by multiplex PCR and SNP array/qPCR. However, all mutations were confirmed by Sanger sequencing and the large deletion was confirmed by qPCR. All the patients in this study were analysed in the previous study of Bonnet et al. [5].

In six patients $(37,5 \%)$, the parents were asymptomatic carriers of Usher genes. The mean BCVA for the group was
0.57 (decimal scale), ranging from 0.1 to 1.0 (SD, 0.32) (Table 1).

\section{SS-OCT results}

The SS-OCT results showed involvement of the ILM (hyperreflective points or waviness) in nine eyes $(28.1 \%)$. MPCs and CMO were detected in $15(46.9 \%)$ and $8(25 \%)$ eyes, respectively. By contrast, ERM was not observed in any of the eyes.

In the analysis of the macular area, we analysed twelve $12.0 \mathrm{~mm}$ radial cuts centred on the fovea. The following abnormalities in the external retinal layers were reported: ELM loss or disruption (27 eyes; 84.4\%); disruption of the Myoid zone (27 eyes; 84.4\%); ISeZ disruption (28 eyes; $87.5 \%$ ), and loss of the OS (29 eyes; 90.6\%). The damage of the RPE was divided according to the loss of the different layers: PhaZ (30 eyes; 93.8\%), RPEmel (29 eyes; 90.6\%) and RPEmitz (0 eyes; 0\%) (Fig. 1).

On the other hand, the same parameters in the subfoveal area were analysed, showing the following results: ELM loss or disruption (4 eyes; $12.5 \%$ ); disruption of the Myoid zone (6 eyes; 18.8\%); disruption of the ISeZ (6 eyes; $18.8 \%$ ), and loss of the OS (16 eyes; $50 \%$ ). The damage of the RPE was divided according to the loss of the different layers: PhaZ (7 eyes; 21.9\%), RPEmel (7 eyes; $21.9 \%$ ) and RPEmitz (0 eyes; 0\%).

Eight eyes (25\%) presented hyperreflective alterations in the vitreous cavity. It must be emphasized though that none 
Fig. 1 SS-OCT scans of the fovea of four patients affected by USH, showing the most representative qualitative retinal and choroidal alterations. In the inner layers, the hyperreflective points $(\mathbf{a}, \mathbf{c})$ and waviness in $\operatorname{ILM}(\mathbf{b}, \mathbf{d})$ are marked with an arrow. Alterations in other inner layers in the form of MPCs $(\mathbf{a}, \mathbf{d})(*)$ or CMO (c) are also shown $(* *)$. The absence of the ELM-IS/OS-OS/RPE complex in the extrafoveal region of the external layers can be seen in all of these images, except for c, which shows the eye of a patient with substantial $\mathrm{CMO}$ leading to the disappearance of all external layers in the subfoveal area. Image d shows important hyperreflective condensations of the vitreous $(+)$. In the choroid, the presence of finer alterations such as thinning of Sattler's layer (a) and the presence of hyperreflective foci $(\mathbf{c}, \mathbf{d})$ can be seen (

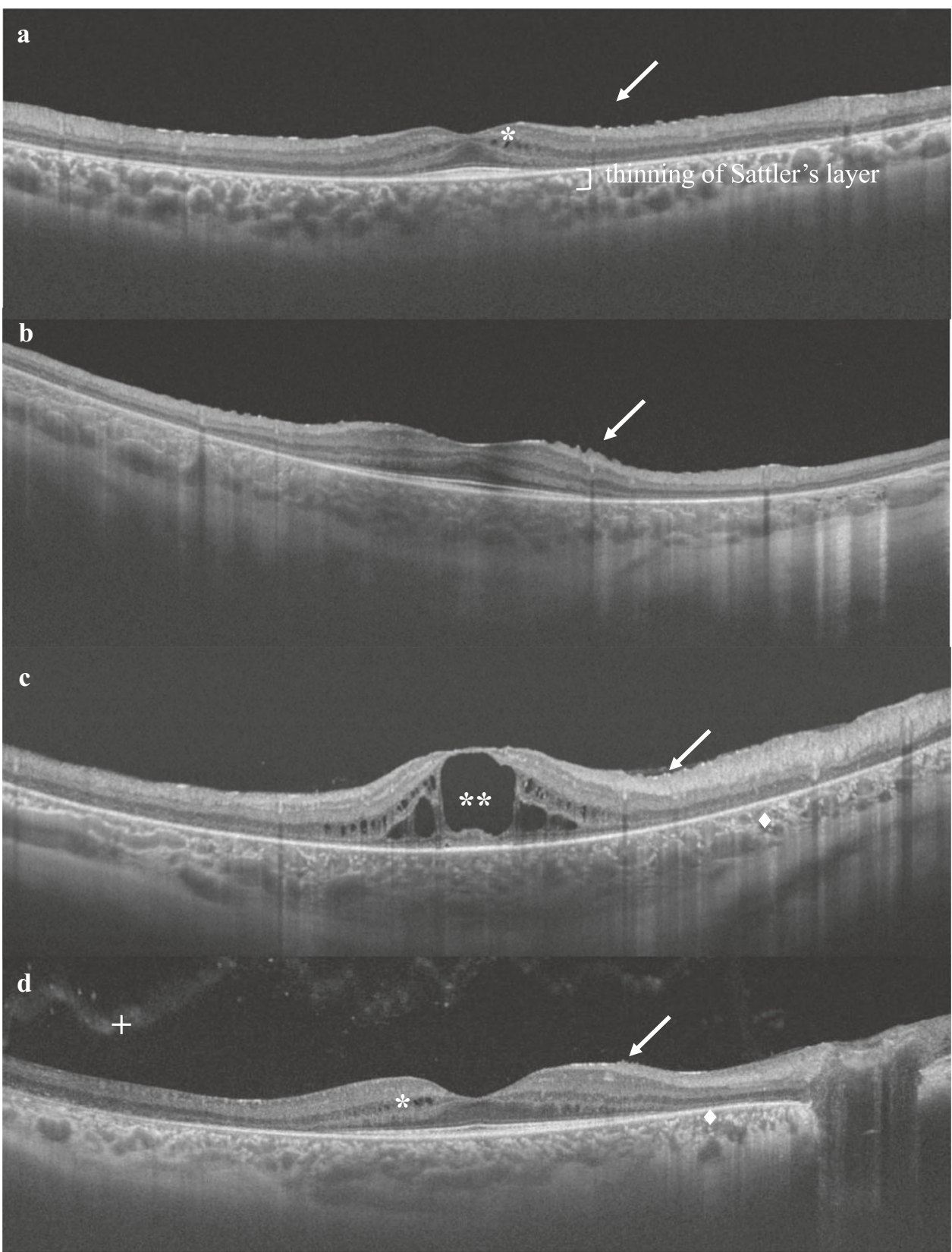

of the patients had vitreous cells in the ophthalmological examination (Fig. 2).

If we perform a general analysis of the state of the photoreceptors and the RPE, considering the damage in any of its segments, we observe the following results: at the macular area photoreceptors and RPE loss is $93.8 \%$ in both cases. Whereas if we focus the analysis in the subfoveal area we observe photoreceptors loss in $50 \%$ of the cases and RPE damage in $21.9 \%$ (Table 3 ).

The following choroidal alterations were observed: hyperreflective foci (25\% of eyes); thinning/absence of Sattler's layer (15.6\%); suprachoroidal hyporeflective space $(9.4 \%)$; and generalized thinning $(3.1 \%)$.
A univariate analysis was performed to assess possible correlations between qualitative OCT findings in the subfoveal area and BCVA. That analysis showed that ELM layer integrity and a preserved Myoid and Ellipsoid zones of photoreceptor layer were associated with better BVCA. In patients presenting absent ELM, the mean BCVA was 0.35 versus 0.5 in patients with normal $\operatorname{ELM}(p=0.024)$. Similarly, the mean BCVA for patients with normal versus disrupted/absent myoid and ellipsoid zone was, respectively, 0.52 and $0.32(p<0.001)$. No significant association was found between BCVA and any of the following: ILM alterations, the presence or absence of CMO or MPCs, alterations in the OS layer or damage in the RPE segments. 
Fig. 2 SS-OCT scans of four patients affected by USH,

showing the most representative external layers alterations in the subfoveal area: a Disruption of outer layers in the macular area, preservation of them in the subfoveal area. b Loss of OS layer of the photoreceptors. c Loss of all the photoreceptor segments: MLE, myoid zone, ellipsoid zone and OS. d Loss of all the photoreceptor segments and also inner segments of RPE (loss of PhaZ and RPEmel and RPEmit preservation)

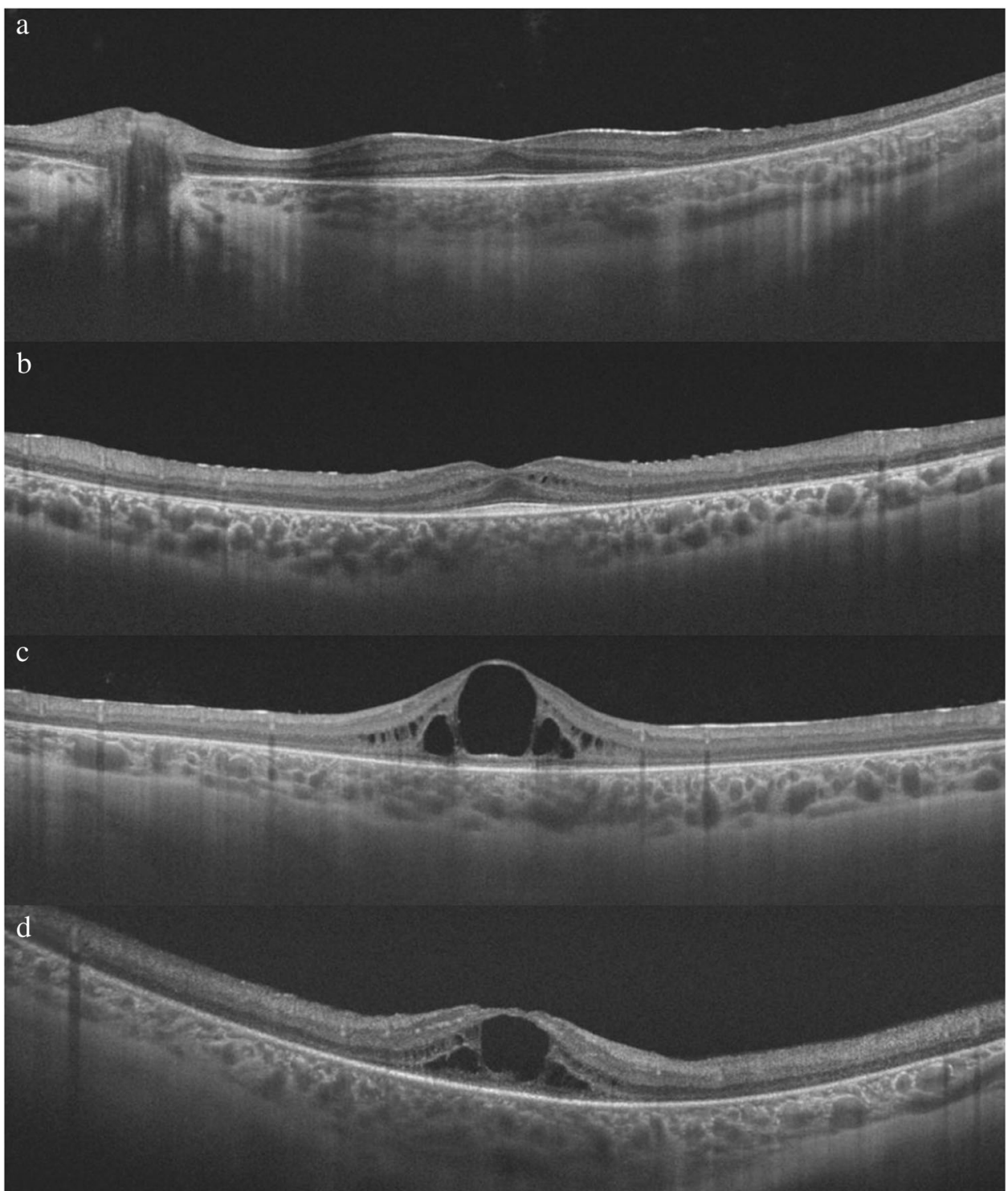

Likewise, we found no association between BCVA and qualitative choroidal alterations.

To control for confounding factors, we performed a multivariate regression analysis. On that analysis, only disruption or absence of the myoid and ellipsoid zones remained independently associated with a lower BCVA (R2 $=0-47 ; t=-4.04 ; p=0.001)$.

Finally, to better understand the pathogenesis of macular oedema in these patients, we evaluated the correlations between CMO and ILM and between CMO and the absence/disruption of any segment of photoreceptors in the subfoveal area. That analysis showed that CMO was significantly correlated with the presence of alterations in the photoreceptor segments $(p=0.013$; correlation coefficient, 0.433 ). By contrast, there was no statistically significant correlation between the presence of CMO and ILM abnormalities $(p=0.827)$.

\section{Discussion}

The main aim of the present report was to study the qualitative retinal alterations detected by SS-OCT in a group of paediatric patients with MYO7A-associated USH1. The most common qualitative abnormality in the retina of the macular area was external layers damage, including ELM loss or disruption (84.4\% of eyes), ISeZ disruption (28 eyes; $87.5 \%$ ) and loss of the OS (29 eyes; $90.6 \%$ ) The damage of the different segments of RPE was: 93.8\% PhaZ and 90.6\% RPEmel and 0\% RPEmitz.

The presence of CMO was significantly correlated with the presence of alterations in the photoreceptor segments. However, on the multivariate analysis, the only variables that were independently associated with decreased BCVA were disruption or absence of the myoid and ellipsoid zones. Overall, it can be speculated that the 
Table 3 Qualitative retinal alterations in macular area

Retinal alterations observed

Frequency and percentage (eyes, $n=32$ )

\begin{tabular}{ll}
\hline ILM (altered) & $9(28.1 \%)$ \\
ERM (present) & 0 \\
MPCs (present) & $15(46.9 \%)$ \\
CMO (present) & $8(25 \%)$ \\
ELM (absent) & $27(84.4 \%)$ \\
Myoid zone (absent) & $27(84.4 \%)$ \\
ISeZ (absent) & $28(87.5 \%)$ \\
OS (absent) & $29(90.6 \%)$ \\
PhaZ (absent) & $30(93.8 \%)$ \\
RPEmel (absent) & $29(90.6 \%)$ \\
RPEmitz (absent) & 0 \\
\hline
\end{tabular}

ILM internal limiting membrane, ERM epiretinal membrane, $M P C$ retinal micropseudocysts, $C M O$ cystoid macular edema, ELM external limiting membrane, IsEZ ellipsoid zone, OS cone outer segments, PhaZ phagosome zone, RPEmel melanosome zone, RPEmitz mitocondria zone

pathophysiologic basis for $M Y O 7 A$-associated USH in early stage disease could be alterations to the outer retinal layers (particularly the photoreceptors) and RPE. Plausibly, these outer layer alterations could be responsible for changes in the inner retinal layers-such as CMO.

OCT imaging can provide valuable data to better understand the alterations induced by USH and to better characterize the various stages of this disease. Prior studies using spectral-domain (SD)-OCT in patients with USH or nonsyndromic RP have reported contradictory results [6-13]. Several different factors could account for these differences, including the type of OCT used, the disease stage, patient age and race, and the USH type.

Testa et al. [7] retrospectively evaluated 134 USH patients. Of these, 42 were diagnosed with USH1 (mean age, $34.4 \pm 17.0$ years) and 91 with USH2 (mean age, 36.2 \pm 13.0 years). The OCT findings revealed the presence of macular abnormalities in 126 of the 268 eyes (47.0\%), with the most common abnormalities as follows: ERM (51 eyes; $19.0 \%$ ), CMO (42 eyes; $15.7 \%$ ), vitreomacular traction (38 eyes; $14.2 \%$ ), and lamellar macular hole (7 eyes; $2.6 \%)$. In that study, macular abnormalities, particularly CMO, were more common in patients with USH1 than in patients with USH2. Sliesoraityte et al. [8] used SD-OCT imaging to evaluate CMO in 76 USH patients (mean age, $42 \pm 14$ years), finding cystic macular lesions in $37 \%$ of the eyes overall and in 45 and $29 \%$, respectively, of the patients with MYO7A (the most common gene in USH1) or USH2A (the most common gene in USH2). Although the authors found some differences between the MYO7A and USH2A groups, these were not statistically significant [8].
Walia et al. performed OCT in 38 patients diagnosed with USH2 (mean age, 38 years), detecting the presence of CMO in 13 cases $(34.2 \%$ ) [9]. Tsilou et al. evaluated 81 patients diagnosed with either USH1 $(n=32$; mean age, 33 years) or USH2 ( $n=34$; mean age, 42 years), finding that cystic foveal lesions were more common in the USH1 group [10].

Numerous studies have been carried out to evaluate macular abnormalities in nonsyndromic RP, revealing wide variability in the prevalence of macular abnormalities. In one study, Grigoropoulos et al. evaluated 42 eyes (21 patients) with RP, finding that CMO and ERM were present, respectively, in $19 \%(n=8)$ and $64.3 \%(n=27)$ of the eyes [11]. However, other authors have found a much lower prevalent of macular abnormalities in patients with RP. Hagiwara et al. used OCT to study 622 eyes from 323 patients with RP, detecting CMO in 34 eyes (5.5\%) and ERM in only 4 eyes [12]. Between these two extremes, a cohort study by Triolo et al., who used SD-OCT to examine 176 eyes from 90 patients with RP, found that the most common retinal alterations were ILM thickening (118 eyes; 67\%) and ERM (48 eyes; 27.3\%). In that study, CMO and MPCs were detected in only $12.5 \%$ and $18.2 \%$ of eyes, respectively [13].

The present study is, to our knowledge, the first to use SS-OCT to assess the presence of retinal abnormalities in a cohort of paediatric patients diagnosed with USH1. Importantly, cystic macular abnormalities (15 eyes with MPCs and 8 eyes with CMO) were detected in more than two-thirds $(71,9 \%)$ of the eyes, a significantly higher percentage than reported in other series. By contrast, none of our patients presented ERM, even though this alteration was the most common change observed in other series. These findings might support the hypothesis that ERM is more common in USH2 patients than in USH1. We found that ERM was less common in USH1 than in nonsyndromic RP, whereas the presence of CMO was more closely associated with USH1 than with nonsyndromic RP and USH2. These findings indicate that USH2 and nonsyndromic RP seem to present similar structural damage patterns, whereas patients with USH1 tend to present a broader range of macular abnormalities. However, larger studies are necessary to determine whether these findings are age related or due to genetically-determined conditions (i.e., Usher type); in this regard, our patient cohort was younger than other published studies and, based on the available evidence, CMO seems to be closely associated with younger age, whereas ERM is more common in older patients [6-13].

The main cause of cystic alterations in the macula is not clear. Kim et al. [14] evaluated a total of 266 eyes-133 of which were from patients with RP (mean age, $47.5 \pm 18.1$ years)—finding that vitreomacular interface abnormalities (VMIAs) were present in $42.7 \%$ of the eyes with RP (they 
did not evaluate the ILM). In that study, VMIAs were significantly more common in eyes with CMO than in those without CMO (64.2\% versus $36.8 \%$; $P<0.001)$; by contrast, the IS/OS junction was better preserved in patients without CMO. Those authors did not assess other outer layers. Triolo et al. [13]. observed ILM thickening in $67 \%$ of RP eyes and ERM in 27.3\%. Those authors found that the IS/OS in the foveal region seems to be independent of the association with an evident $\mathrm{CMO}$, contrary to the report by Kim et al. who found that CMO was strongly correlated with IS/OS disruption [14].

One novel finding of the current study was the presence of ILM alterations-in the form of hyperreflective points or undulations-that have not been previously associated with ERM or ILM thickening in USH patients (Fig. 1). Importantly, these ILM abnormalities were present in nearly onefourth of the eyes in our sample. The most common macular alteration in our patient cohort was outer layer damage, consisting of IS/OS disruption/absence in $>90 \%$ of the eyes, absence of the ELM in $>80 \%$, and OS/RPE disruption of more than two-thirds of patients. CMO was significantly correlated with alterations at the OS/RPE junction, but not with ILM or IS/OS abnormalities. Surprisingly, while both Triolo et al. [13] and Kim et al. [14] analysed the IS/OS junction, neither assessed the OS/RPE junction.

Other authors have performed biomicroscopic ocular evaluations in patients with RP, finding that vitreous alterations-including floating cottonball-like condensations, fibrillary degeneration, pigmentary or non-pigmentary vitreal particulation, and posterior vitreous detachment - are common in these patients [15]. In our study, close to $30 \%$ of the patients showed evidence of vitreous opacities on the OCT scan.

Our findings suggest that USH1 may initially affect the outer retinal layers, particularly the photoreceptors, and that alterations in these layers promote the development of CMO, regardless of the specific abnormalities present in the vitreomacular interface. We hypothesize that degeneration of the photoreceptors might induce a nonspecific inflammatory response, but the mechanisms leading to photoreceptor cell death are still unclear. Previous studies suggested that photoreceptor cell loss in RP is driven by apoptosis [16], but more recent reports indicate that other cell death pathways, such as the activation of cysteineproteases, lysosomal proteases, autophagy and complementmediated lysis, could be involved [17].

Another relevant question in this study is: Is MYOA7retinopathy in a USH1 a photoreceptor-first or RPE-first disease? Due to the fact that none of the study participants had RPEmitz lesions, it can be speculated that MYO7Aretinopathy results in photoreceptor loss as a primary event. In the progression of the disease, it might involve the inner segments of the RPE (PhaZ and RPEmel). The fact that we have not found RPEmitz lesions in any eye is the main argument for this hypothesis.

There are two locations of mitochondria cells in the retina: ellipsoid zone and RPE mitochondria zone. The mitochondria are critical organelles for cell function and survival. Their primary roles are adenosine triphosphate (ATP) production, control of cellular metabolism and regulation of apoptosis (programmed cell death) [4]. Our results show $87.5 \%$ of eyes with ellipsoid zone loss in the macular area, but no single eye with RPEmitz loss.

Of the various retinal alterations described in this report, only damage at the myoid and ellipsoid zones of photoreceptors were independently associated with a decrease of the BCVA. This finding is consistent with the report by Battaglia et al., who found that changes to the ELM and OS/ RPE layers are independently associated with BCVA in patients with RP [18].

The findings of the present study indicate that the physiopathologic basis of early stage USH1 may be alterations in the outer retinal layers, especially the photoreceptors. Consequently, clinicians should consider monitoring the condition of photoreceptors during follow up, as this could permit the early detection and treatment of pathologic changes. Indeed, the status of the photoreceptors and the inner segments of the RPE (PhaZ and RPEmel) could be important parameters to monitor to ensure proper follow-up of USH patients. The main limitation of this study is the cross-sectional design, which does not allow us to determine the time course of the changes to the photoreceptors. More studies are needed to better characterize the correlation between CMO and damage to the outer layers and to better understand the cell death pathways involved in these alterations.

\section{Summary}

\section{What was known before}

- Which were the retinal abnormalities in adult patients with Usher syndrome detected by OCT, specially in outer layers.

\section{What this study adds}

- The differences of these abnormalities in a cohort of paediatric patients with Usher syndrome.

Acknowledgements This work received no funding. We thank the families HEALTH-F2-2010-242013 (TREATRUSH). We also thank Bradley Londres for editing and improving the use of English in this document and Dr. Anne Kurtenbach for her critical review and comments of this paper. 


\section{Compliance with ethical standards}

Conflict of interest The authors declare that they have no conflict of interest.

Publisher's note: Springer Nature remains neutral with regard to jurisdictional claims in published maps and institutional affiliations.

\section{References}

1. Milam AH, Li ZY, Fariss RN. Histopathology of the human retina in retinitis pigmentosa. Prog Retin Eye Res. 1998;17: 175-205.

2. Boughman JA, Vernon MSK. Usher syndrome: definition and estimate of prevalence from two high-risk populations. J Chronic Dis. 1983;36:595-603.

3. Narayan DS, Wood JPM, Chidlow G, Casson RJ. A review of the mechanisms of cone degeneration in retinitis pigmentosa. Acta Ophthalmol. 2016;94:748-54.

4. Cuenca N, Ortuño-Lizarán I, Pinilla I. Cellular characterization of OCT and outer retinal bands using specific immunohistochemistry markers and clinical implications. Ophthalmology. 2018;125: 407-22.

5. Bonnet C, Riahi Z, Chantot-Bastaraud S, Smagghe L, Letexier M, Marcaillou C, et al. An innovative strategy for the molecular diagnosis of Usher syndrome identifies causal biallelic mutations in 93\% of European patients. Eur J Hum Genet. 2016; 24:1730-8.

6. Jin P, Zou H, Zhu J, Xu XUN, Jin J, Chang TAC, et al. Choroidal and retinal thickness in children with different refractive status measured by swept-source optical coherence tomography. Am J Ophthalmol. 2016;168:164-76.

7. Testa F, Melillo P, Rossi S, Marcelli V, Benedictis D, Colucci R. et al. Prevalence of macular abnormalities assessed by optical coherence tomography in patients with Usher syndrome. Ophthalmic Genet. 2018;39:17-21.
8. Sliesoraityte I, Peto T, Mohand-said S, Sahel JA. Novel grading system for quantification of cystic macular lesions in Usher syndrome. Orphanet J Rare Dis. 2015;10:157.

9. Walia S, Fishman GA, Hajali M. Prevalence of cystic macular lesions in patients with Usher II syndrome. Eye. 2008;23:1206-9.

10. Tsilou ET, Rubin BI, Caruso RC, Reed GF, Pikus A, Hejtmancik $\mathrm{JF}$, et al. Usher syndrome clinical types I and II: Could ocular symptoms and signs differentiate between the two types? Acta Ophthalmol Scand. 2002;80:196-201.

11. Grigoropoulos VG, Emfietzoglou J, Nikolaidis P, Chatzistefanou KVJ, Theodossiadis GPTP. Optical coherence tomography findings in patients with retinitis pigmentosa and low visual acuity. Ophthalmic Surg Lasers Imaging. 2010;41:35-9.

12. Hagiwara A, Yamamoto S, Ogata K, Sugawara T, Hiramatsu A, Shibata M, et al. Macular abnormalities in patients with retinitis pigmentosa: prevalence on OCT examination and outcomes of vitreoretinal surgery. Acta Ophthalmol. 2011;89:e122-5.

13. Triolo G, Pierro L, Parodi MB, De Benedetto U, Gagliardi M, Manitto MP, et al. Spectral domain optical coherence tomography findings in patients with retinitis pigmentosa. Ophthalmic Res. 2013;50:160-4.

14. Kim YJ, Joe SG, Lee D, Lee JY, Kim J. Correlations between spectral-domain OCT measurements and visual acuity in cystoid macular edema associated with retinitis pigmentosa. Invest Ophthalmol Vis Sci. 2013;54:1303-9.

15. Vingolo EM, Giusti C, Forte ROP. Vitreal alterations in retinitis pigmentosa: biomicroscopic appearance and statistical evaluation. Ophthalmologica. 1996;210:104-7.

16. Chang G, Hao Y, Wong F, Carolina N. Apoptosis: final common pathway of photoreceptor death in rd, rds, and rhodopsin mutant mice. Neuron. 1993;11:595-605.

17. Lohr HR, Kuntchithapautham K, Sharma AK. Multiple, parallel cellular suicide mechanisms participate in photoreceptor cell death. Exp Eye Res. 2006;83:380-9.

18. Battaglia PM, La SC, Triolo G, Riccieri F, Pierro L, Gagliardi M, et al. Correlation of SD-OCT findings and visual function in patients with retinitis pigmentosa. Graefes Arch Clin Exp Opththalmol. 2016;254:1275-9. 